\title{
Growth and stability of SmS-TaS 2 nanotubes studied by XAFS and DAFS methods
}

\author{
A. Khadiev' ${ }^{1}$, S.M. Bhyrappa ${ }^{2}$, R. Tenne ${ }^{2}$, D. Novikov ${ }^{1}$ \\ ${ }^{I}$ DESY Photon Science, Deutsches Elektronen-Synchrotron DESY, 22607 Hamburg, ${ }^{2}$ Weizmann Institute of Science, Rehovot, Israel
} azat.khadiev@desy.de,sreedharamb@weizmann.ac.il

It is known, that during the nanotube (NT) synthesis additional unwanted phases often occur (platelets, fullerenes, amorphic content, etc.)[1] and it is very hard or even impossible to disentangle the information about the NTs from the data achieved by macroscopic methods. The purpose of this study is to show the advantages of the DAFS (Diffraction Anomalous Fine Structure) spectroscopy technique in analysis of NT powders containing a sufficient number of unwanted phases. This technique, based on measurement of the diffraction peak intensity in the vicinity of the X-ray absorption edge, could bring additional spectroscopic information about the tubes in the powder mixture. Contrary to conventional XAFS (X-ray absorption Fine Structure) spectroscopy, DAFS allows to measure the XAFS-like signal from a certain phase or crystallographic site separately by choosing the proper diffraction peak [2].

NTs have several diffraction features, that distinguish them from the conventional 3D crystals and single 2D layers, that are based on the same structural units/layers (Carbon nanotubes vs Graphite, etc). Due to the lack of the out-of-plane symmetry diffraction patterns of the NTs usually doesn't show the distinct reflections of the $h 0 l$ and $0 \mathrm{kl}$ type, that distinguish them from the bulk particles; contrary to the single 2D layers the multilayered NTs show the reflections of $00 l$ type due to diffraction from the basal planes of the NT [3]. These $00 l$ and $h k 0$ reflections can be used to get information about the NTs using the DAFS technique from NT raw powder.

In order to understand the growth and stability of $\mathrm{SmS}_{-} \mathrm{TaS}_{2} \mathrm{NT}$ a set of NT powders were synthesized by chemical vapor transport method (CVT) [2] at different temperatures $(800,825,850,875,900,925,975,1050 \mathrm{C})$. It was found from XRD that a small amount of $\mathrm{Ta}_{1.2} \mathrm{~S}_{2}$ and $\mathrm{Ta}_{1.08} \mathrm{~S}_{2}$ present in all samples, intensity of $\mathrm{Sm}_{2} \mathrm{Ta}_{3} \mathrm{~S}_{2} \mathrm{O}_{8}$ and $\mathrm{SmTaO}_{4}$ phases starts to grow prominently at $875 \mathrm{C}$ temperature. From XRD and electron microscopy studies it was also found that the NT abundancy falls down with temperature. XAFS-like spectra derived from NT 002 and 026 reflections DAFS also (Fig. 1) shows temperature dependence: the intensity of Ta L3 'white' line decrease with temperature. Such dependence of 'white' line intensity on NT abundancy can be explained by the difference in interaction of $\mathrm{SmS}$ and $\mathrm{TaS}_{2}$ layers in bulk $\mathrm{SmS}_{-} \mathrm{TaS}_{2}$ crystals and $\mathrm{SmS}^{-\mathrm{TaS}_{2}} \mathrm{NT}$. In the bulk SmS-TaS $\mathrm{S}_{2}$ crystals (usually defined as $\left.(\mathrm{SmS})_{1.19} \mathrm{TaS}_{2}\right)$ it was found that $\mathrm{SmS}$ layer act as a donor of electrons for the $\mathrm{TaS}_{2}$ part [4]. In the NT due to the curvature of the layer, the number of the SmS units is smaller than in the bulk crystals, thus SmS part donate less electrons. Therefore, in NT there should be more Ta $5 \mathrm{~d}$ band vacancies in comparison to bulk, thus providing more intense Ta L3 'white' line in XANES spectra.

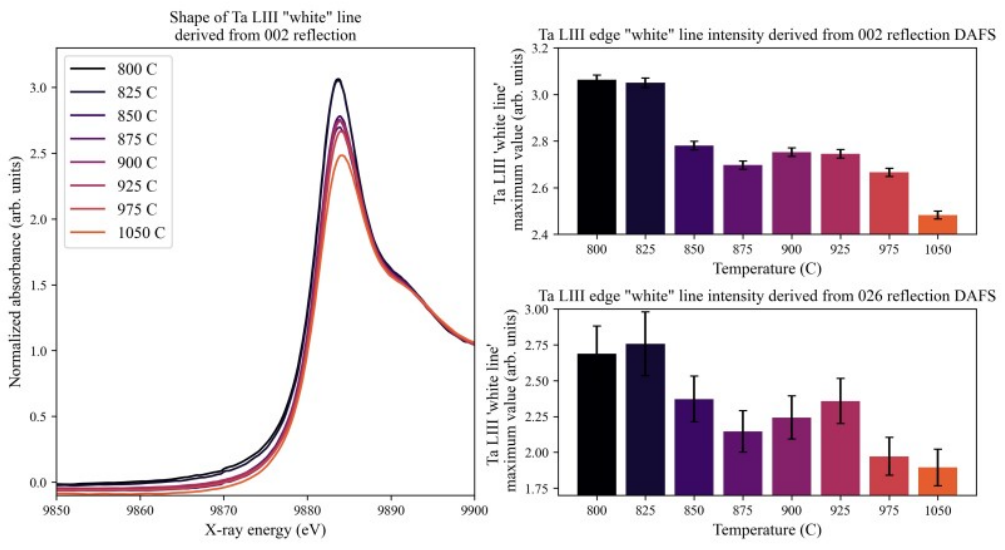

Figure 1. XAFS-like spectra derived from 002 reflection (left) DAFS, 'white' intensity derived from 002 (right top) and 026 (right bottom) reflections DAFS.

[1] Serra, M. et al. (2020). Appl. Mater. Today. 19, 100581.

[2] Kawaguchi, T., Fukuda, K. \& Matsubara, E. (2017). J. Phys. Condens. Matter. 29, 113002.

[3] Khadiev, A. \& Khalitov, Z. (2018). Acta Crystallogr. Sect. A Found. Adv. 74, 233.

[4] Wiegers, G. A., Meetsma, A., Haange, R. J. \& de Boer, J. L. (1991). J. Less Common Met. 168, 347.

\section{Keywords: DAFS; XAFS; misfit nanotubes; inorganic nanotubes}

Acta Cryst. (2021), A77, C998 